\title{
Treatment Options for People with Posttraumatic Stress Disorder (PTSD)
}

A recent update of a systematic review, supported by PCORI through a research partnership with $A H R Q$, informs clinicians on psychological and pharmacological treatments for PTSD in adults. ${ }^{1}$ The review reports on 207 articles from 193 studies published before 2018, updating a 2013 review.

osttraumatic Stress Disorder (PTSD) affects about $6 \%$ of US adults. It is more common in groups including women, younger people, and those who did not complete high school or who have lower incomes. ${ }^{2}$ PTSD can affect military personnel serving in combat, but it may also develop after a person experiences or witnesses intimate partner violence, sexual violence, physical abuse or assault, a motor vehicle crash, natural disaster, violent crime, or other traumatic event.
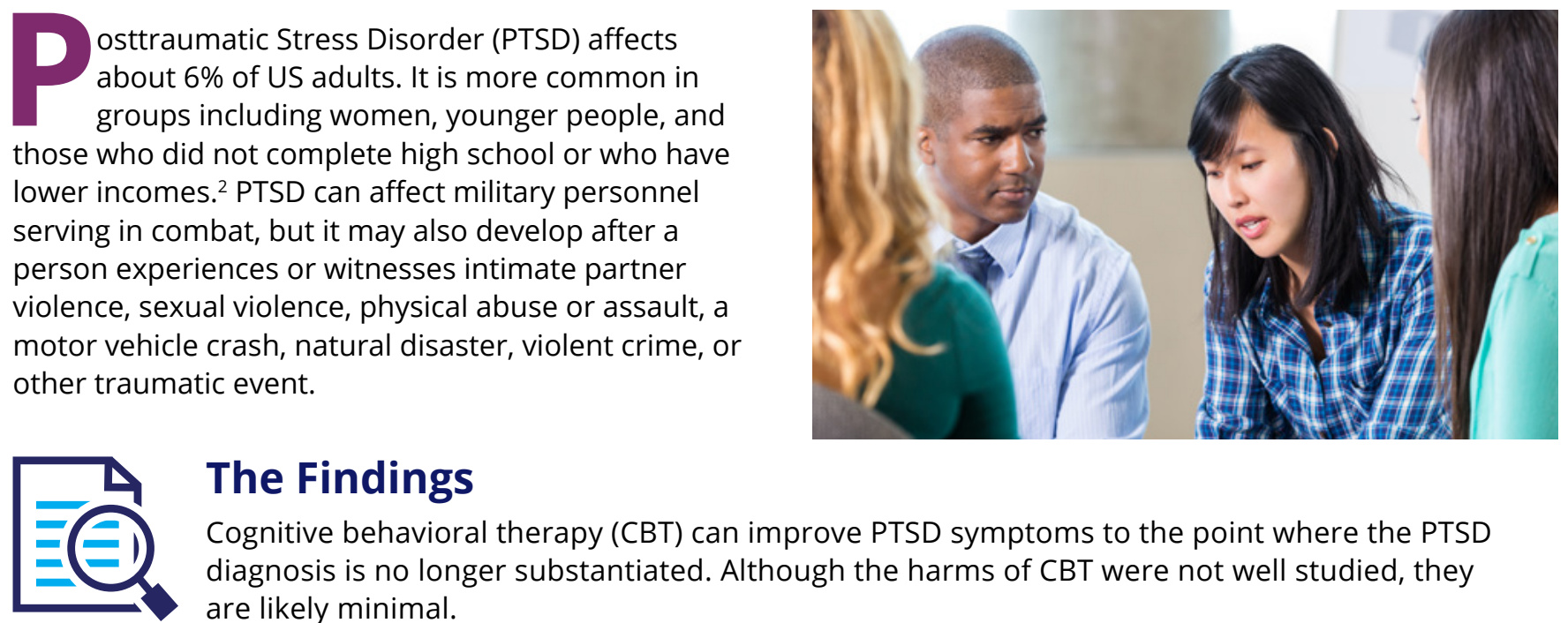

\section{The Findings}

Cognitive behavioral therapy (CBT) can improve PTSD symptoms to the point where the PTSD diagnosis is no longer substantiated. Although the harms of CBT were not well studied, they are likely minimal.

\section{Cognitive Behavioral Therapy}

- A specific form of CBT called exposure therapy and a group of other treatments that included elements of CBT had high strength of evidence (SOE) of effectiveness for improving PTSD symptoms. The SOE increased from the 2013 review.

- Other forms of CBT (cognitive processing therapy, cognitive therapy, and narrative exposure therapy) and eye movement desensitization and reprocessing (EMDR) had moderate SOE for effectiveness of treating PTSD.

\section{Medication}

- Three medications (fluoxetine, paroxetine and venlafaxine*) had moderate SOE of effectiveness for treating PTSD. The SOE for these medications having side effects such as nausea was low to medium. Side effects vary by medication.

- Due to mixed findings in the existing evidence, the SOE of the effectiveness of both sertraline and topiramate for treating PTSD was reduced from moderate to low in the updated review.

*Fluoxetine - brand names include Prozac ${ }^{\circledR}$, Prozac $^{\circledR}$ weekly, Rapiflux ${ }^{\circledR}$, Sarafem ${ }^{\circledR}$, and Selfemra ${ }^{\circledR}$

*Paroxetine - brand names include Brisdelle ${ }^{\circledR}$, Paxil ${ }^{\circledR}$, Paxil $^{\circledR} \mathrm{CR}$, and Pexeva ${ }^{\circledR}$

*Venlafaxine - brand names include Effexor ${ }^{\circledR}$ and Effexor ${ }^{\circledR}$ XR 


\section{Professional Guidelines}

Clinical practice guidelines are available from the following organizations:

- American Psychological Association $^{3}$

- U.S. Department of Veterans Affairs/U.S. Department of Defense ${ }^{4}$

\section{Psychological Treatment Modalities with High or Moderate Evidence of Effectiveness}

\author{
Cognitive Behavioral \\ Therapy (CBT)
}

Exposure Therapy
A broad category of therapies that includes components of both cognitive and behavioral therapies. In using CBT to treat patients with PTSD, the therapist may use exposure, cognitive restructuring, and/or various coping skills.

Involves confronting feared stimuli and using imagery to extinguish the conditioned emotional response (usually anxiety) to traumatic stimuli.

\section{SOURCES}

1. Forman-Hoffman V, Middleton JC, Feltner C, et al. Psychological and Pharmacological Treatments for Adults With Posttraumatic Stress Disorder: A Systematic Review Update. This systematic review was supported by PCORI through a research partnership with AHRQ. Rockville, MD: AHRQ Publication No. 18-EHC011-EF; 2018. Washington, DC: PCORI Publication No. 2018-SR-01; 2018. DOl: https://doi.org/10.23970/ AHRQEPCCER207.

2. Goldstein RB, Smith SM, Chou $S P$, et al. The Epidemiology of DSM-5 Posttraumatic Stress Disorder in the United States: Results from the National Epidemiologic Survey on Alcohol and Related Conditions-III. Soc Psychiatry Psychiatr Epidemiol. 2016;51(8):1137-48.

3. American Psychological Association. Clinical Practice Guideline for the Treatment of PTSD. Washington, DC: American Psychological Association; 2017. https://www.apa.org/ptsdguideline/ptsd.pdf

4. U.S. Department of Veterans Affairs. VA/DOD Clinical Practice Guideline for the Management of Posttraumatic Stress Disorder and Acute Stress Disorder. Version 3.0. Washington, DC: U.S. Department of Veterans Affairs, Department of Defense; 2017. https://www.healthquality.va.gov/ guidelines/MH/ptsd/

\section{Cognitive Processing Therapy}

Increases awareness of dysfunctional or distorted trauma-related thoughts and helps patients correct or replace those thoughts with more adaptive or rational thoughts and beliefs.
Combines exposure therapy with the concurrent induction of rapid, intermittent eye movements believed to help reprogram brain function to resolve the emotional impact of trauma.

Adapts exposure therapy to meet the unique needs of those exposed to war and torture.

\section{Communicating with Patients about PTSD}

A patient version of this evidence update is available at: www.pcori.org/ new-evidence-PTSD. You can use it when talking with patients about treatment options.

Current evidence does not suggest which patients would benefit most from particular treatments. Clinicians should consider evidence-based guidelines and patient preference in selecting a treatment. 